\title{
GPS based child care system using RSSI technique
}

\begin{abstract}
In today's society, missing child cases increases rapidly. Child care is a critical issue in solving this epidemic. Various systems and methods have been implemented in solving this crisis. In this paper, the global position system (GPS) based child care system is proposed to solve this problem. This system consists of two nodes; child and parent nodes, each carried by the child and parent respectively. The child node consists of a simple circuit that has a Bluetooth module and a GPS receiver. The parent node is a mobile device that has Bluetooth connection. The GPS technology helps to determine the exact position of the child. The parent node receives the location of the child and displays the information on a designed map using a GUI program that is embedded into the mobile device. Besides the location, the distance between parent and child can also be determined using Bluetooth connection. A data from received signal strength indicator (RSSI) is extracted out from the Bluetooth connection and is used as vital information in determining the distance between parent and child. An alert is triggered when the distance between the parent and child is far apart for a certain range. The proposed system proves to be useful as a locator that could locate missing and wandering child for the parents.
\end{abstract}

Keyword: GPS; Bluetooth; RSSI; Child care system 